\title{
Effects of long-term soft contact lens wear on the corneal thickness and corneal epithelial thickness of myopic subjects
}

\author{
YULIN LEI ${ }^{1,2}$, XIUYUN ZHENG ${ }^{2}$, JIE HOU ${ }^{2}$, BAOZENG XU² and GUOYING MU ${ }^{3}$ \\ ${ }^{1}$ School of Medicine, Shandong University, Jinan, Shandong 250012; ${ }^{2}$ Department of Ophthalmology, \\ Jinan Mingshui Eye Hospital, Zhangqiu, Shandong 250200; ${ }^{3}$ Department of Ophthalmology, \\ Provincial Hospital Affiliated with Shandong University, Jinan, Shandong 250021, P.R. China
}

Received April 14, 2014; Accepted August 6, 2014

DOI: $10.3892 / \mathrm{mmr} .2014 .2964$

\begin{abstract}
To perform safe and successful corneal refractive surgery on myopic patients, corneal thickness (CT) and corneal epithelial thickness (CET) must be accurately measured. Numerous individuals with myopia wear soft contact lenses (SCLs) for the correction of visual acuity but may subsequently undergo corneal refractive surgery. The aim of the present study was therefore to investigate the effects of long-term SCL wear on the CT and the CET of myopic subjects in order to guarantee the safety and accuracy of subsequent corneal refractive surgeries. Fifty-six subjects prepared to receive refractive surgery at Jinan Mingshui Eye Hospital (Zhangqiu, China) from April to July 2013 were included in the study. CT and CET were measured in subjects immediately following discontinued SCL wear (group I, 56 eyes), and subsequently following $>$ two weeks of discontinued SCL wear (group II, 56 eyes). Ninety-four subjects with no history of corneal contact lens wear were enrolled as a control group. The CT and CET were measured at positions with a radius of $0.0-1.0$, 1.0-2.5 (divided into eight quadrants) and 2.5-3.0 $\mathrm{mm}$ (divided into eight quadrants) away from the corneal center using the RTVue-100 Fourier-domain anterior segment optical coherence tomography system. A significant decrease in the CT of the subjects in group II was observed, compared with that of group I and the control group $(\mathrm{P}<0.05)$. A significant decrease was observed in the CET of groups I and II compared with that of the control group $(\mathrm{P}<0.05)$. Following discontinuation of SCL wear, CET increased. However, the increased CET was unable to reach the normal range exhibited by the control group. Edema and thinning of the corneal stroma, as well as thinning of the corneal epithelium were observed in groups I
\end{abstract}

Correspondence to: $\mathrm{Dr}$ Guoying $\mathrm{Mu}$, Department of Ophthalmology, Provincial Hospital Affiliated with Shandong University, 324 Jingwuweiqi Road, Jinan, Shandong 250021, P.R. China

E-mail: sdjneye2014@163.com

Key words: myopia, corneal thickness, corneal epithelial thickness, anterior segment optical coherence tomography and II. In conclusion, it was proposed that in clinical practice, for myopic patients following long-term SCL wear, CT and CET should be determined $\geq$ two weeks following discontinuation of SCL wear, once a stable CT and CET are obtained.

\section{Introduction}

Corneal thickness (CT) is frequently used as a potential determinant of the safety of corneal refractive surgery (1). Accurate determination of CT prior to corneal refractive surgery has been proposed for the determination of the corneal ablation rate, as well as for the evaluation of postoperative refractive stability and safety (1). Thus, measurement of the CT prior to the initiation of corneal refractive surgery is crucial.

The wearing of soft contact lenses (SCLs) has been reported to affect the normal physiological structure of the cornea, in particular the CT. Prior to corneal refractive surgery, a large variance was observed in the CT of individuals following discontinuation of long-term SCL wear. By contrast, the effects of corneal contact lenses on CT largely depended on the duration of wear, mode and the quality of the lenses (2). A study by Liu and Pflugfelder (3) using the Orbscan corneal topography system reported that long-term wear of corneal contact lenses decreased overall CT. Conversely, an instant increase in CT was observed in short-term SCL wearers (4). Optical coherence tomography (OCT), a technique for performing high-resolution cross-sectional imaging, is frequently used as an alternative to Orbscan tomography as it has demonstrated higher reproducibility and accuracy (5).

Following advances in corneal biomechanics, corneal surface ablation techniques including Epipolis laser in situ keratomileusis (EPI-LASIK) and transepithelial photorefractive keratectomy (T-PRK) have been frequently used in corneal refractive surgery (6). Additionally, ultra-LASIK techniques such as Femtosecond laser-assisted Sub-Bowman's Keratomileusis (SBK) have also been used (7).

The corneal epithelium, as the first protective barrier of the cornea, has a vital function in the prevention of pathogenic microorganism invasion (8). The corneal epilthelium also functions as an essential optical transduction and refraction media with a marked capacity for regeneration and repair (9). It has been proposed that the measurement of corneal epithelial thickness (CET) is significant in the early diagnosis of 
os

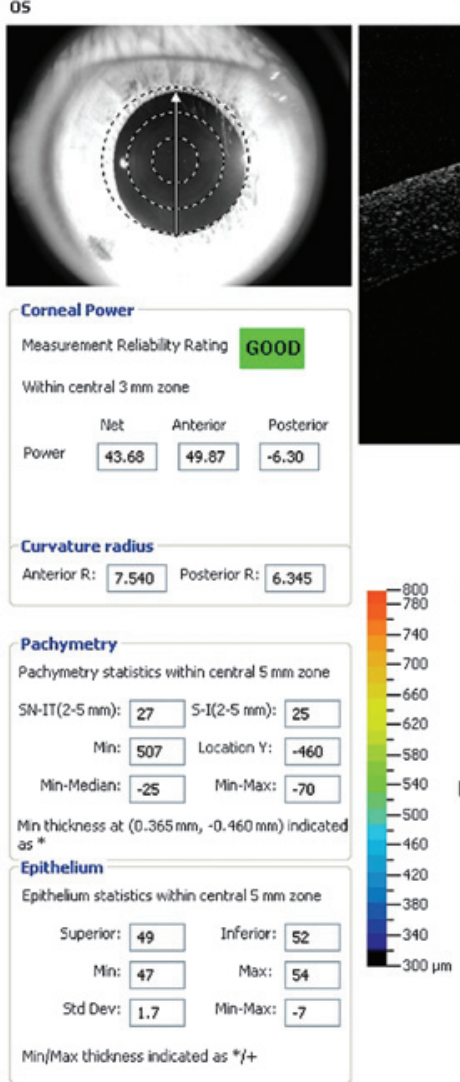

Pachymetry + CPwr $\quad S S I=47.6$

Figure 1. Data obtained using anterior segment optical coherence tomography examination.

keratoconus and in epithelial healing following corneal refractive surgery (10-12). Although studies have been performed to measure the CET, measurements were limited to the central region of the cornea $(11,13,14)$. It is therefore necessary to measure the whole CET, in order to investigate the characteristics of the thickness across the entire cornea.

In the present study, Fourier-domain anterior segment OCT was used to evaluate the CT and CET in SCL wearers prior to being subjected to corneal refractive surgery. The present study aimed to elucidate the normal ranges and distribution characteristics of CT and CET in myopic subjects, and therefore provide guidance for ophthalmologists in order to improve the safety, predictability and stability of corneal refractive surgeries.

\section{Subjects and methods}

Subjects. Prospective patients for refractory surgery at the excimer laser treatment center at Jinan Mingshui Eye Hospital (Zhangqiu, China) from April to July 2013 were included in this study. The inclusion criterion was the wearing of SCLs for $>$ two years. Patients with ocular diseases were excluded from the present study. A total of 56 right eyes of 56 subjects were enrolled. CT and CET were measured prior to refractory surgery in subjects immediately following discontinued SCL wear (group I, 56 eyes), and subsequently following discontinued SCL wear for >two weeks (group II, 56 eyes). Ninety-four eyes of 94 subjects with no history of wearing corneal contact lenses were enrolled as a normal control group. Informed consent was obtained from all subjects. The present study was consistent with the Declaration of Helsinki, and was approved by the Ethics Committee of Zhangqiu Mingshui Hospital (Zhangqiu, China).

Anterior segment OCT examination. Imaging of the anterior segment was performed using the RTVue-100 Fourier-domain anterior segment OCT system (Optovue Inc., Fremont, CA, USA) by an experienced ophthalmologist. The OCT was performed based on the optical Fourier-domain imaging technique (15). Briefly, subjects were required to watch the indicator light under natural conditions. On this basis, Pachymetry and corneal power scanning modes were selected for the scanning and the scanning diameter was set at $6 \mathrm{~mm}$. Eight meridians were selected with 1024 axial scans per meridian. The full procedure was repeated five times. The center of the pupil was set as the focus site. To eliminate the potential effects caused by the dodging of the eyelash or eyelid during CT determination, subjects were required to open their eyes widely. RTVue-CAM software (Version 6.11; Optovue Inc.) automatically processed the OCT scan, provided the pachymetry (corneal thickness) map and indicated CT and CET ranges at the positions with radii of $0.0-1.0,1.0-2.5$ (divided into eight quadrants) and 2.5-3.0 $\mathrm{mm}$ (divided into eight quadrants) from the corneal center (Fig. 1). This procedure was performed in triplicate.

Statistical analysis. All values are expressed as the mean \pm standard deviation. Data analysis was performed using SPSS 19.0 software (International Business Machines, Armonk, NY, USA). Student's t-test was used for the inter-group comparison 


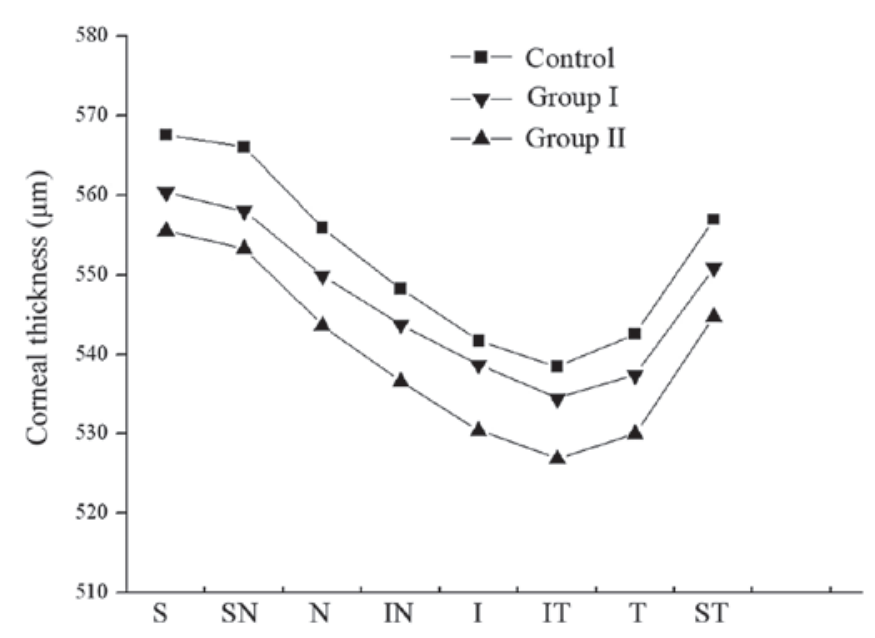

Figure 2. Corneal thickness distribution in regions with a radius of $1.0-2.5 \mathrm{~mm}$ from the corneal center in myopic subjects. T, temporal; N, nasal; $\mathrm{S}$, superior; I, inferior.

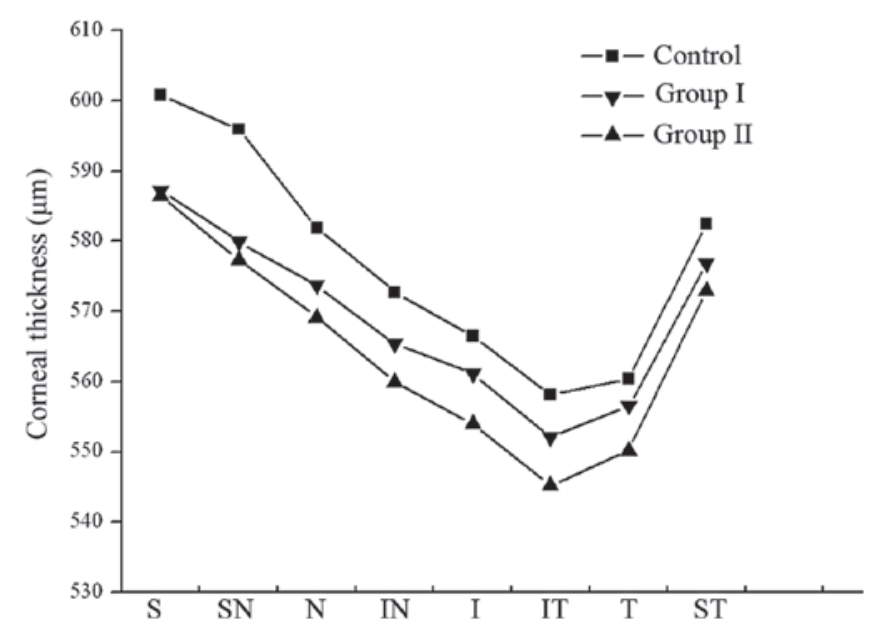

Figure 3. Corneal thickness distribution in regions with a radius of 2.5-3.0 mm from the corneal center in myopic subjects. T, temporal; N, nasal; S, superior; I, inferior.

of CT and CET. Paired sample t-test was performed for the CT and CET between two subgroups. Spearman's correlation analysis was applied to analyze the correlation between CT and CET. $\mathrm{P}<0.05$ was considered to indicate a statistically significant difference between values.

\section{Results}

Subject information. In total, 94 eyes of 94 subjects (male, 71; female, 23) were enrolled in the control group. The age range of subjects in the control group was 18-32 years with a mean age of $21.26 \pm 5.01$ years. In the study group, 56 eyes of 56 subjects (male, 24; female, 32 ) were enrolled. The age range of subjects in the study group was 18-35 years with a mean age of $22.02 \pm 4.52$ years. In the study group, the duration of SCL wear was 2-9 years with a mean of $3.86 \pm 2.15$ years.

Correlation between the central corneal thickness (CCT) and epithelial thickness of the central cornea. In the control group,
Table I. CT and CET in regions with a radius of $0-1 \mathrm{~mm}$ from the corneal center in myopic subjects.

\begin{tabular}{lll}
\hline Groups & \multicolumn{1}{c}{ CT } & CET \\
\hline Control & $533.56 \pm 27.42$ & $55.05 \pm 2.71$ \\
Group I & $529.31 \pm 27.94$ & $49.78 \pm 3.82^{\mathrm{a}}$ \\
Group II & $521.45 \pm 25.99^{\mathrm{ab}}$ & $51.18 \pm 2.22^{\mathrm{ab}}$ \\
\hline
\end{tabular}

${ }^{\mathrm{a}} \mathrm{P}<0.05$, compared with the control group; ${ }^{\mathrm{b}} \mathrm{P}<0.05$, compared with group I.

the mean CCT was $533.56 \pm 27.42 \mu \mathrm{m}$ in a position with a radius of $1 \mathrm{~mm}$ from the central cornea, while the mean epithelial thickness of the central cornea was $55.05 \pm 2.71 \mu \mathrm{m}$. A small positive correlation was observed between the CCT and the epithelial thickness of the central cornea $(r=0.237, P=0.022$; Table I).

The mean CCT of group I was $529.31 \pm 27.94 \mu \mathrm{m}$, which indicated no significant difference compared with that of the control group $(\mathrm{t}=0.903, \mathrm{P}=0.368)$. In group II, the mean CCT was $521.45 \pm 25.99 \mu \mathrm{m}$. Compared with the control group, a reduced CCT $(2.27 \%)$ was observed in group II $(\mathrm{t}=2.689$, $\mathrm{P}=0.008)$. Furthermore, a significant difference was noted between the CCT of group I and that of group II $(\mathrm{t}=5.859$, $\mathrm{P}<0.001)$.

A statistically significant difference was observed in the central thickness of the corneal epithelium in group I compared with that of the control group $(49.78 \pm 3 \mu \mathrm{m}$ vs $55.05 \pm 2.71 \mu \mathrm{m}$, $\mathrm{t}=8.986, \mathrm{P}<0.001)$. In addition, there was a statistically significant difference in the central thickness of the corneal epithelium in group II compared with that of the control group $(51.18 \pm 2.22 \mu \mathrm{m}$ vs $55.05 \pm 2.71 \mu \mathrm{m}$; $\mathrm{t}=9.447, \mathrm{P}<0.001)$. Moreover, a significant difference was observed between the central thickness of the corneal epithelium of group I and that of group II ( $\mathrm{t}=-3.683, \mathrm{P}=0.001)$.

Effects of SCLs on CT. The CTs of the control and study groups are presented in Tables I and II. For the CT in a location with a radius of $1.0-2.5$ or $2.5-3.0 \mathrm{~mm}$ from the corneal center, the minimal CT was observed in the inferior temporal area and the maximal $\mathrm{CT}$ was observed in the superior area (Tables I and II). The CT was decreased in the study groups. In group II, the CT in the location with a radius of $1.0-2.5 \mathrm{~mm}$ from the corneal center was significantly decreased compared with that obtained prior to discontinuation of SCL wear $(\mathrm{P}<0.05)$. For the $\mathrm{CT}$ in a location with a radius of $2.5-3.0 \mathrm{~mm}$ from the corneal center, there was no statistical difference in the $\mathrm{CT}$ in the superior region and the nasal region prior to and following discontinuation of SCL wear $(\mathrm{P}>0.05)$. However, significant differences were observed in the CT in other regions (Tables II and III; Figs. 2 and 3).

Effects of SCLs on CET. In all corneal regions, the CET at a position with a radius of 1.0-2.5 $\mathrm{mm}$ and 2.5-3.0 $\mathrm{mm}$ from the corneal center indicated that the CET was comparatively thicker in the inferior section and thinner in the superior section. In the study group, the CET was decreased in all 

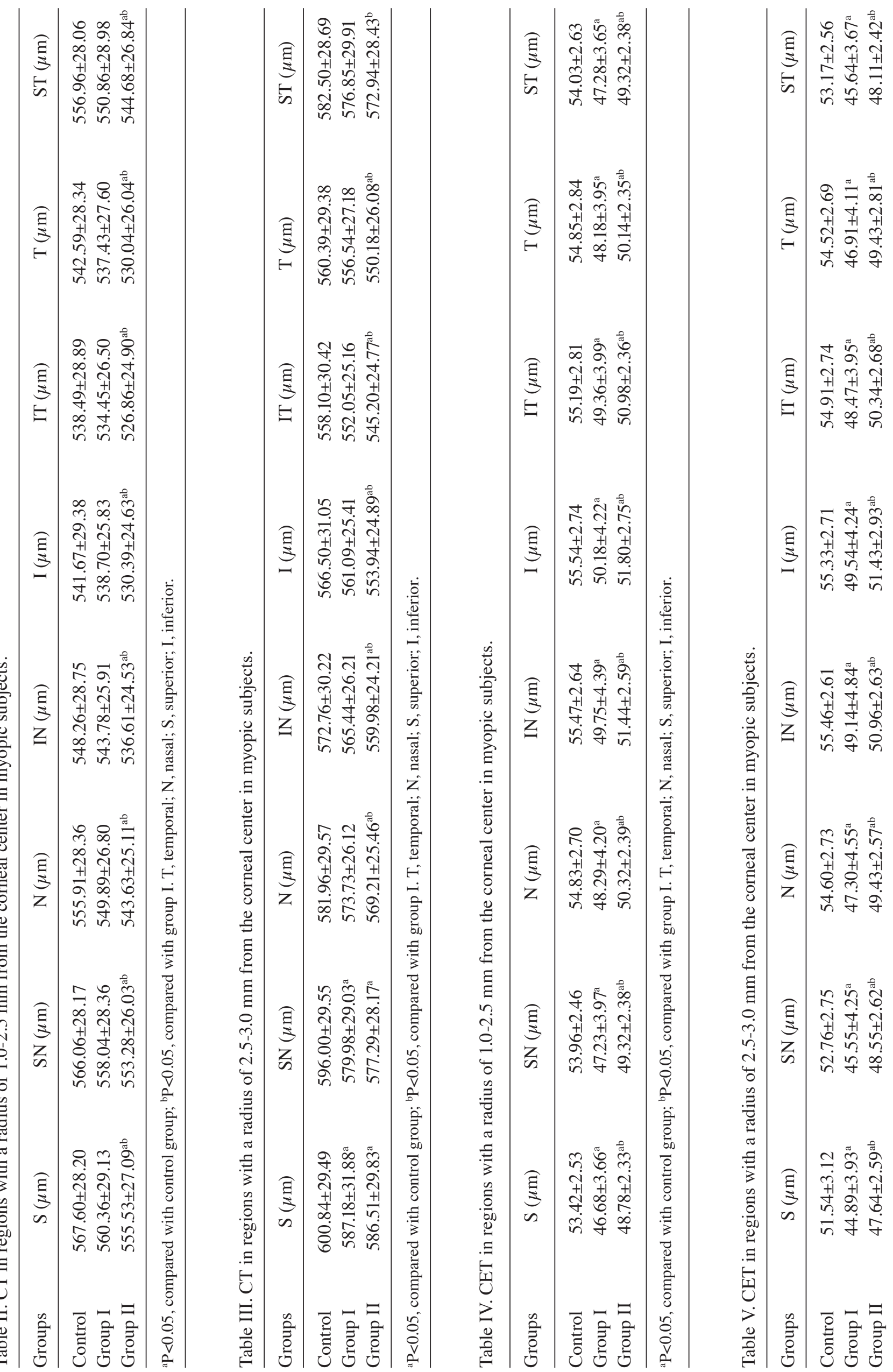

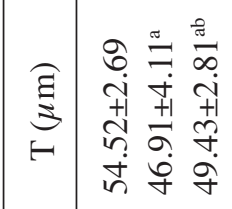

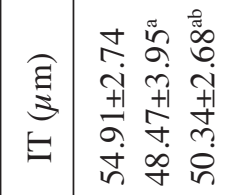

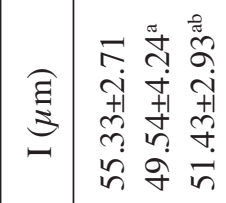

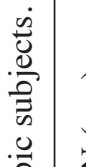

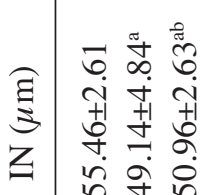

:

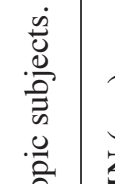

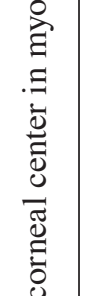

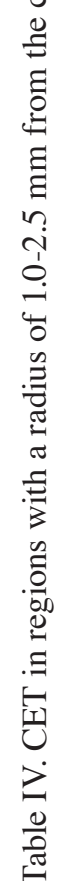

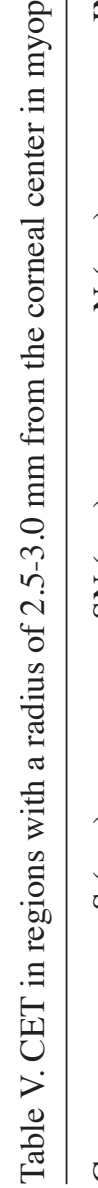

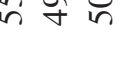

๓

है

乙

官

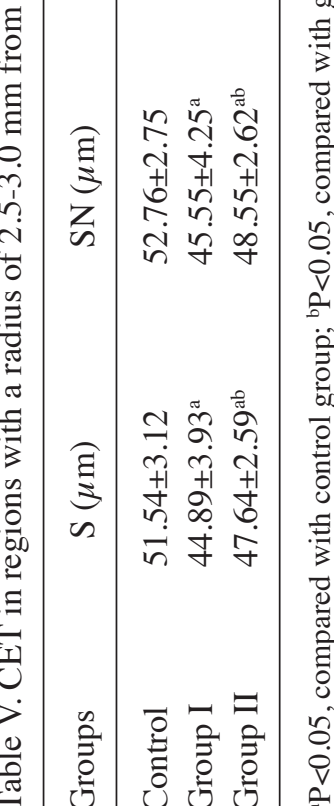




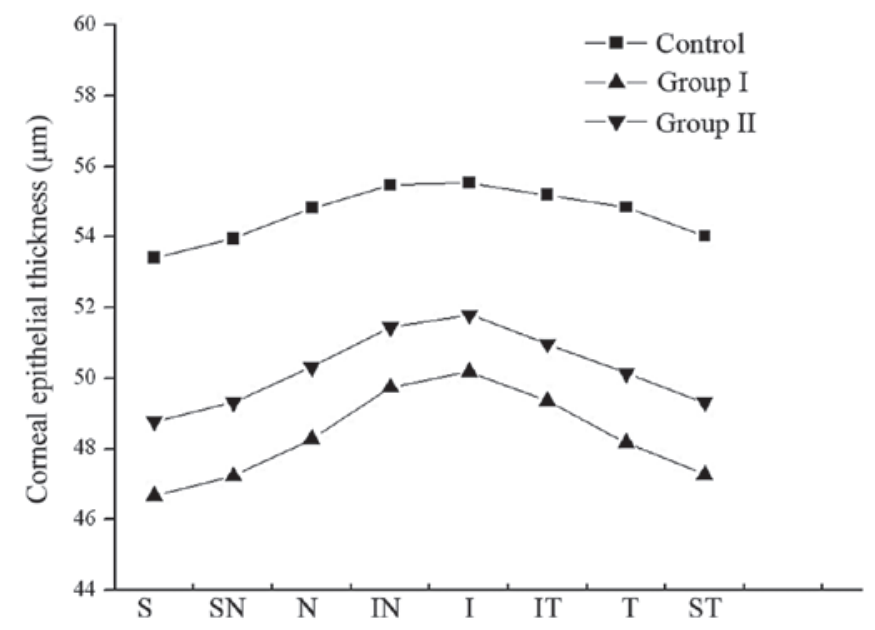

Figure 4. Corneal epithelial thickness distribution in regions with a radius of 1.0-2.5 mm from the corneal center in myopic subjects. T, temporal; $\mathrm{N}$, nasal; $\mathrm{S}$, superior; I, inferior.

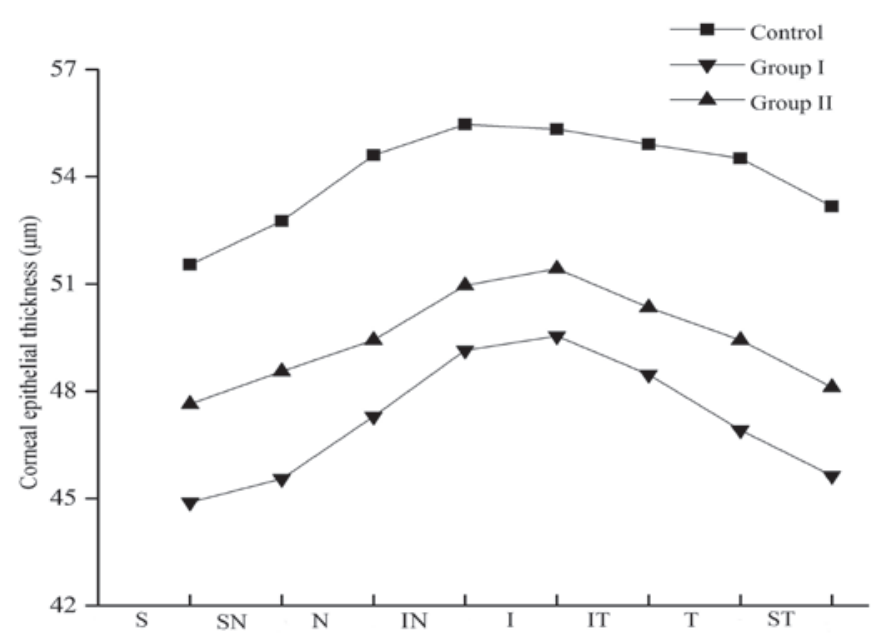

Figure 5. Corneal epithelial thickness distribution in regions with a radius of 2.5-3.0 mm from the corneal center in myopic subjects. T, temporal; $\mathrm{N}$, nasal; $\mathrm{S}$, superior; I, inferior.

regions. However, the CET increased following discontinuation of SCL wear, though the thickness was unable to return to the normal range. Differences in CET were statistically significant in these corneal regions $(\mathrm{P}<0.05$; Tables IV and V; Figs. 4 and 5).

\section{Discussion}

SCLs provide a straightforward option for the correction of visual acuity whilst maintaining a satisfactory aesthetic appearance and are therefore popular amongst individuals with myopia, particularly teenagers (16). Corneal refractive surgery provides an alternative option for the correction of visual acuity of myopic patients. According to clinical data, numerous myopia patients with experience of wearing SCLs have resorted to corneal refractive surgery to obtain satisfactory visual acuity (17). In order to perform safe and successful corneal refractive surgery, CT and CET must be accurately measured so that the appropriate type of surgery can be selected, cutting parameters can be determined and postoperative refractive stability and corneal remodeling can be monitored. Therefore, evaluating the effects of SCLs on CT and CET is essential in order to guarantee the safety and accuracy of corneal refractive surgeries.

To date, measurement of CT has largely depended on ultrasound and optical techniques. For example, the development of instruments including optical coherence tomography $(10,11,18,19)$, ultrasonic thickness gauges (13) and confocal microscopes $(14,20)$ have contributed to innovations in CT determination.

Anterior segment OCT, a novel technique for quantitative analysis of ocular parameters, is able to accurately measure $\mathrm{CT}$ and CET and has the advantages of requiring no contact and being non-invasive as well as simpler to perform. Anterior segment OCT is divided into time-domain OCT and Fourierdomain OCT according to the measurement principles utilized.

In the present study, Fourier-domain OCT with a scanning frequency of 26,000 sweeps/sec was used for the determination of CT and CET. The axial resolution was elevated to $3 \mu \mathrm{m}$. Moreover, CT and CET were automatically calculated by the installed software, which may have reduced the potential errors caused by conventional manual software. The CT and CET data were presented in regions and quadrants.

In a previous study, Hashemi et al (21) determined that the time required to reach corneal stability following discontinued daily wearing of SCLs was a two-week contact lens-free period. Nourouzi et al (22) reported that a 2-15 day cessation of SCL wear was required to eliminate the corneal edema associated with SCL wear. Furthermore, a stable CT was achieved in $74 \%$ of patients within one week, and in a further $26 \%$ of patients during the second week. The present study hypothesized that a stable CT was achieved within two weeks of discontinued SCL wear, however, individual variation could not be excluded.

Corneal oxygen uptake has a crucial role in the energy metabolism and maintenance of transparency of the cornea $(23,24)$. Corneal oxygen uptake is mainly dependent upon the exchange with the outside air or blood vessels located in the palpebral conjunctiva $(23,25,26)$. Under normal conditions, corneal oxygen uptake is obstructed by eye-closing in the evening, which induces a disorder of the sodium-potassium pump and leads to corneal edema (27). On this basis, corneal edema occurs with a rate of 5-6\% (27); however, is completely reversed in the waking state as sufficient oxygen is obtained $(25,26)$.

Corneal oxygen uptake is obstructed due to SCL wear when eyes are open or closed (28). This disruption of corneal oxygen supply may lead to physiological metabolic disorders, including corneal edema and increased corneal thickness. In the present study, the CT in group II was thicker than that of group I $(\mathrm{P}<0.05)$, which confirmed the presence of corneal edema during long-term SCL wear. In addition, the thickness of the corneal epithelium in group II was greater than that of group I, which demonstrated that the chronic corneal edema induced by SCL wear occurred mainly in the corneal stroma.

A study reported a significant decrease in the CET of individuals with long-term or occasional SCL wear compared with that of control subjects (18). In a retrospective analysis, pachymetry measurement was performed in order to compare the CT of spectacle-wearing control eyes with those of SCL wearers and rigid gas-permeable contact lens wearers (29). 
It was concluded that there was a significant reduction in the CT of full-time contact lens-wearing subjects. In the present study, a clear decrease was observed in the CT of subjects in group II compared with that of subjects in the control group and group I. These results appear to confirm that the CT was decreased following long-term SCL wear. Taking the $\mathrm{CT}$ in these groups into consideration, it was concluded that decreased CT and concurrent edema were identified in myopic subjects following long-term SCL wear.

The potential mechanisms of corneal edema and thinning are varied. For example, Wang et al (30) reported an increased CT in subjects with short-term SCL wear, using the OCT technique, and thus demonstrated the presence of corneal edema. Furthermore, chronic corneal edema was frequently identified in long-term SCL wearing subjects, which led to biochemical changes in the corneal stroma and a significant reduction in the CT (31.32). It has additionally been reported that enhanced tear osmotic pressure was present in SCL wearers, leading to thinning of the whole cornea (33). A further potential mechanism is that long-term SCL wear may induce reversible corneal epithelial compression, necrosis and collapse of the corneal epithelium, or cell apoptosis of the corneal stroma (3). In subjects with long-term and/or prolonged SCL wear, several factors have been reported to be associated with the cellular apoptosis of cornea and CT reduction, including changes in the microenvironment of the ocular surface, chronic microlesion in the corneal tissues and chronic hypoxia (34).

Extensive studies have focused on the measurement of CT, and in particular CCT; however, it is necessary to measure the CET of various regions for the accurate analysis of CET distribution $(35,36)$. To date, the thickness evaluation has been performed in the peripheral region of the corneal epithelium and the majority of the data were obtained from the inferior and/or superior region of the cornea $(2,10,22,29)$. A previous study aimed to measure the CET in the vertical and horizontal meridians of the cornea and demonstrated that the corneal epithelial thinning was topographically uniform $(19,37)$. By contrast, Simon et al (38) suggested that no layer of uniform thickness was formed by the corneal epithelium over Bowman's layer. Furthermore, the changes of the CET distribution mainly resulted from the verified corneal surface power, and the orientation of the astigmatism axis. Using the RTVue-100 system, the results of the present study indicated that the CET was not evenly distributed, as the CET in the inferior cornea was comparatively thicker than that of the superior cornea.

A previous study indicated a $6 \%$ decrease in the CET of subjects with long-term SCL wear (39). Consistent with the results by Liesegang (39), the CET obtained in group I was markedly thinner than that in the control group. It was hypothesized that the CET reduction observed may be associated with corneal epithelial microlesions caused by mechanical irritation of the epithelial surface during long-term SCL wear (40-42). Amongst SCL wearers, dissection and denaturation of corneal epithelial cells were observed using scanning electron microscopy. Simultaneously, a mild separation was noticed in the intercellular space of the corneal epithelial cells observed through a transmission electron microscope. In the presence of corneal epithelial microlesion, permeability of corneal epithelial cells was increased as a result of decreased corneal ion pump function, which resulted in dehydrolysis. Therefore, chronic hypoxia has a marked influence on physiological function (40).

In the present study, a significant increase in CET was demonstrated in group II compared with that of group I $(\mathrm{P}<0.05)$. It was hypothesized that this was mainly due to the regeneration of the corneal epithelium. Following discontinuation of SCL wear, the associated hypoxia and mechanical irritation were eliminated and therefore, partial self-repair and reproduction may be induced in the corneal epithelium.

In the present study, the changes in CT and CET were analyzed in long-term SCL-wearing subjects. The results of the present study demonstrated edema and thinning of the corneal stroma in myopia long-term SCL-wearing subjects. Furthermore, thinning of the corneal epithelium was noted in these subjects.

In conclusion, based on the results of the present study it was proposed that in clinical practices, for myopic patients with long-term SCL wear, determination of CT and CET should be performed two weeks following discontinuation of SCL wear, once a stable CT and CET are obtained. On this basis, surgical strategy may be reliably established. Greater attention should be paid to patients with reduced CT, irregular cornea, and severe astigmatism, as well as those with corneal ectasia or keratoconus to avoid postoperative secondary keratoconus.

\section{References}

1. Reinstein DZ, Srivannaboon S, Archer TJ, Silverman RH, Sutton H and Coleman DJ: Probability model of the inaccuracy of residual stromal thickness prediction to reduce the risk of ectasia after LASIK part I: quantifying individual risk. J Refract Surg 22: 851-860, 2006.

2. Walline JJ, Gaume A, Jones LA, Rah MJ, Manny RE, Berntsen DA, Chitkara M, Kim A and Quinn N: Benefits of contact lens wear for children and teens. Eye Contact Lens 33: 317-321, 2007.

3. Liu Z and Pflugfelder SC: The effects of long-term contact lens wear on corneal thickness, curvature, and surface regularity. Ophthalmology 107: 105-111, 2000.

4. Bonanno JA and Polse KA: Central and peripheral corneal swelling accompanying soft lens extended wear. Am J Optom Physiol Opt 62: 74-81, 1985.

5. Fujimoto JG, Pitris C, Boppart SA and Brezinski ME: Optical coherence tomography: an emerging technology for biomedical imaging and optical biopsy. Neoplasia 2: 9-25, 2000.

6. Stillitano I, Yamazaki E, Melo LA Jr, Bottos J and Campos M: Wavefront-guided refractive surgery results of training-surgeons. Arq Bras Oftalmol 73: 323-328, 2010.

7. Sia RK, Coe CD, Edwards JD, Ryan DS and Bower KS:Visual outcomes after Epi-LASIK and PRK for low and moderate myopia. J Refract Surg 28: 65-71, 2012.

8. Bergmanson JP: Histopathological analysis of the corneal epithelium after contact lens wear. J Am Optom Assoc 58: 812-818, 1987.

9. Lu L, Reinach PS and Kao WW: Corneal epithelial wound healing. Exp Biol Med (Maywood) 226: 653-664, 2001.

10. Li Y, Tan O, Brass R, Weiss JL and Huang D: Corneal epithelial thickness mapping by Fourier-domain optical coherence tomography in normal and keratoconic eyes. Ophthalmology 119: 2425-2433, 2012.

11. Haque S, Jones L and Simpson T: Thickness mapping of the cornea and epithelium using optical coherence tomography. Optom Vis Sci 85: E963-E976, 2008.

12. Huang D, Tang M and Shekhar R: Mathematical model of corneal surface smoothing after laser refractive surgery. Am J Ophthalmol 135: 267-278, 2003.

13. Reinstein DZ, Archer TJ, Gobbe M, Silverman RH and Coleman DJ: Epithelial thickness in the normal cornea: three-dimensional display with Artemis very high-frequency digital ultrasound. J Refract Surg 24: 571-581, 2008. 
14. Li HF, Petroll WM, Møller-Pedersen T, Maurer JK, Cavanagh HD and Jester JV: Epithelial and corneal thickness measurements by in vivo confocal microscopy through focusing (CMTF). Curr Eye Res 16: 214-221, 1997.

15. Ramos JL, Li Y and Huang D: Clinical and research applications of anterior segment optical coherence tomography - a review. Clin Experiment Ophthalmol 37: 81-89: 2009.

16. Ehsaei A, Chisholm CM, MacIsaac JC, Mallen EA and Pacey IE: Central and peripheral visual performance in myopes: contact lenses versus spectacles. Cont Lens Anterior Eye 34: 128-132, 2011

17. Lim L and Wei RH: Laser in situ keratomileusis treatment for myopia in a patient with partial limbal stem cell deficiency. Eye Contact Lens 31: 67-69, 2005.

18. Feng Y and Simpson TL: Comparison of human central cornea and limbus in vivo using optical coherence tomography. Optom Vis Sci 82: 416-419, 2005.

19. Wang J, Fonn D, Simpson TL, Sorbara L, Kort R and Jones L: Topographical thickness of the epithelium and total cornea after overnight wear of reverse-geometry rigid contact lenses for myopia reduction. Invest Ophthalmol Vis Sci 44: 4742-4746, 2003

20. Patel SV, McLaren JW, Hodge DO and Bourne WM: Confocal microscopy in vivo in corneas of long-term contact lens wearers. Invest Ophthalmol Vis Sci 43: 995-1003, 2002.

21. Hashemi H, Firoozabadi MR, Mehravaran S and Gorouhi F: Corneal stability after discontinued soft contact lens wear. Cont Lens Anterior Eye 31: 122-125, 2008.

22. Nourouzi H, Rajavi J and Okhovatpour MA: Time to resolution of corneal edema after long-term contact lens wear. Am J Ophthalmol 142: 671-673, 2006.

23. Chen Y, Thompson DC, Koppaka V, Jester JV and Vasiliou V: Ocular aldehyde dehydrogenases: protection against ultraviolet damage and maintenance of transparency for vision. Prog Retin Eye Res 33: 28-39, 2013.

24. Thiel HJ: Epithelium of the cornea and its significance for transparency. Dtsch Med Wochenschr 99: 1630-1635, 1974 (In German)

25. Takatori SC, de la Jara PL, Holden B, Ehrmann K, Ho A and Radke CJ: In vivo oxygen uptake into the human cornea. Invest Ophthalmol Vis Sci 53: 6331-6337, 2012.

26. Weissman BA, Fatt I and Rasson J: Diffusion of oxygen in human corneas in vivo. Invest Ophthalmol Vis Sci 20: 123-125, 1981.

27. Efron $\mathrm{N}$ and Carney LG: Oxygen levels beneath the closed eyelid Invest Ophthalmol Vis Sci 18: 93-95, 1979.

28. Ichijima $\mathrm{H}$, Hayashi $\mathrm{T}$, Mitsunaga $\mathrm{S}$ and Hamano $\mathrm{H}$ : Determination of oxygen tension on rabbit corneas under contact lenses. CLAO J 24: 220-226, 1998.
29. Braun DA and Anderson Penno EE: Effect of contact lens wear on central corneal thickness measurements. J Cataract Refract Surg 29: 1319-1322, 2003.

30. Wang J, Fonn D, Simpson TL and Jones L: The measurement of corneal epithelial thickness in response to hypoxia using optical coherence tomography. Am J Ophthalmol 133: 315-319, 2002.

31. Hutchings N, Simpson TL, Hyun C, Moayed AA, Hariri S, Sorbara L and Bizheva K: Swelling of the human cornea revealed by high-speed, ultrahigh-resolution optical coherence tomography. Invest Ophthalmol Vis Sci 51: 4579-4584, 2010.

32. Efron N, Perez-Gomez I and Morgan PB: Confocal microscopic observations of stromal keratocytes during extended contact lens wear. Clin Exp Optom 85: 156-160, 2002.

33. Cerretani C, Peng CC, Chauhan A and Radke CJ: Aqueous salt transport through soft contact lenses: an osmotic-withdrawal mechanism for prevention of adherence. Cont Lens Anterior Eye 35: 260-265, 2012.

34. McMonnies CW, Chapman-Davies A and Holden BA: The vascular response to contact lens wear. Am J Optom Physiol Opt 59: 795-799, 1982.

35. Kanellopoulos AJ and Asimellis G: Anterior segment optical coherence tomography: assisted topographic corneal epithelial thickness distribution imaging of a keratoconus patient. Case Rep Ophthalmol 4: 74-78, 2013.

36. Zhou W and Stojanovic A: Comparison of corneal epithelial and stromal thickness distributions between eyes with keratoconus and healthy eyes with corneal astigmatism $\geq 2.0 \mathrm{D}$. PLoS One 9: e85994, 2014

37. Pérez JG, Méijome JM, Jalbert I, Sweeney DF and Erickson P: Corneal epithelial thinning profile induced by long-term wear of hydrogel lenses. Cornea 22: 304-307, 2003.

38. Simon G, Ren Q, Kervick GN and Parel JM: Optics of the corneal epithelium. Refract Corneal Surg 9: 42-50, 1993.

39. Liesegang TJ: Physiologic changes of the cornea with contact lens wear. CLAO J 28: 12-27, 2002.

40. Pearlman E, Sun Y, Roy S, Karmakar M, Hise AG, Szczotka-Flynn L, Ghannoum M, Chinnery HR, McMenamin PG and Rietsch A: Host defense at the ocular surface. Int Rev Immunol 32: 4-18, 2013

41. Markoulli M, Papas E, Cole N and Holden B: Corneal erosions in contact lens wear. Cont Lens Anterior Eye 35: 2-8, 2012.

42. Brewitt H: Contact lenses. 2: Contact lens associated infections. Klin Monbl Augenheilkd 211: aA7-A10, 1997 (In German). 